\title{
The State of the Art in Providing Automated FEEDBACK TO OPEN-ENDED STUDENT WORK
}

\author{
Paula Larrondo, Brian Frank, and Julián Ortiz \\ Queen's University \\ \{p.larrondo, brian.frank, julian.ortiz\}@ queensu.ca
}

\begin{abstract}
This article provides a review of the state of the art of technologies in providing automated feedback to open-ended student work on complex problems. It includes a description of the nature of complex problems and elements of effective feedback in the context of engineering education. Existing technologies based on traditional machine learning methods and deep learning methods are compared in light of the cognitive skills, transfer skills and student performance expected in a complex problemsolving setting. Areas of interest for future research are identified.
\end{abstract}

Keywords: Automate feedback, formative feedback, openended complex problem-solving skills, artificial intelligence-based technologies, engineering complex problems.

\section{INTRODUCTION}

Complex problem-solving (CPS) skills and critical thinking are core engineering education requirements $[5$, 31]. Future engineers need to be able to use knowledge to solve complex problems, find innovative solutions to novel problems, create new knowledge, and communicate their findings properly [4]. Jonassen et al. [32] summarized this requirement in a shared workplace appraisal: "practicing engineers are hired, retained, and rewarded for solving problems" (p.139).

The ability to address these future challenges comes by addressing a variety of increasingly complex authentic problems that require those skills [34]. Developing expertise and self-regulation strategies requires frequent practice accompanied by feedback and self-reflection in open-ended work [12], [36], [44]. Students need feedback on their understanding and procedures, but the feedback they receive on how, where, and when they transfer their knowledge to solve a problem is more decisive in developing problem-solving and critical thinking skills [51].

Substantial evidence has been provided on the potential positive impact of Formative Assessment (FA) in effective learning [3], [22], [23], [39], [44], [57], [60]. Formative feedback given as part of FA have several advantages over other types of assessments. Among the most important is that it can be given to students as they are working on a problem, allowing them to clarify and correct misconceptions and improve their thinking and learning quality. Formative feedback also provides instructors with insight into the learning process and has the potential to translate into better pedagogical practices that improve students' learning, motivation, and engagement [2], [51], [54], [57], [60]. To be a valuable instructional strategy, FA has to be adequately designed to be interrelated with instruction, focus on the task, have a clear purpose, be meaningful, and be optimum in timing for the kind of learning. Therefore, designing and giving effective feedback for learning in complex and ill-structured tasks is a demanding multifactorial instructional strategy for instructors. Aside from task-specific requirements, effective feedback needs to consider learning outcomes, appropriate timing, and individual learners' cognitive, metacognitive, and motivational disposition [16].

Educational technologies can be a valuable learning support to provide feedback [58], supplementing feedback provided by instructional teams. Artificial intelligencebased technologies allow students, teachers, and content experts to interact, both synchronously and asynchronously. Through these interactions, feedback can be increased; active learning strengthened; different learning styles accommodated, and personalized, tailored scaffolding and instruction provided.

The purpose of the present work is to examine research done to automate meaningful and multidimensional feedback to open-ended student responses to complex problems. In the next section, we proposed the analytical framework based on Jonassen conceptualization of problem-solving assessment. The following section includes a synthesis of the existing research for different skills to be assessed, including conventional machine learning and deep learning approaches using supervised, unsupervised, and semi-supervised training. Considering that feedback can be given as part of the assessment, the present review included both automated feedback applications as well as automated assessment. This 
research aims to identify the methods and algorithms that could be implemented for an automated feedback system for engineering courses and identify areas where more research is needed.

\section{ANALYTICAL FRAMEWORK}

The present review was done under the conceptual framework proposed by Jonassen for the assessment of problem-solving in the engineering context. In particular, research was classified based on the following elements: (1) understanding the problem through problem schema assessment, (2) student problem-solving performance, (3) cognitive skills and domain knowledge, and (4) argumentative reasoning.

Complex problems can be classified as well-structured or ill-structured [10], though problem complexity is not exclusively a function of the structuredness of a problem. Complexity relates to the number of elements presented in the situation, whether explicit or implicitly presented, how these elements interact and how much they change in time. In addition, how much of this information is available to the student was used by Maker and Schiever to classify problems as close or open-ended [56].

Developing complex problem-solving skills has been the object of extensive studies [4],[8], [13], [18], [19], [25]. Within the western academic corpus exist two broad traditions in what CPS is and how to assess it: the European and North American perspectives. The main difference between the two approaches is that the European perspective focuses on complex computerized laboratory tasks that mimic real-world but non-disciplinary problems. In contrast, the North American tradition focuses on problem-solving in natural knowledge domains which is especially critical for engineering education. Practicing engineers should be trained in a variety of complex problems specific to the discipline, which are not necessarily the same as other scientific disciplines. Accordingly, the present review was done under the theoretical framework proposed by Jonassen in "Engineers as Problem Solvers" [36].

Jonassen [33] adhered to the perspective that CPS depends on the field of application but expands the idea. To develop problem-solving skills, the author emphasizes the need for students to face a variety of ill-structured complex problems rather than well-structured textbook problems with known solution paths and convergent answers. Engineering workplace problems commonly have unclear or multiple solution paths, a high degree of uncertainty, multidisciplinary requirements, conflicting goals, non-engineering constraints, and numerous criteria evaluations of solutions [12], [31], [36]. Therefore, engineering instruction needs to include open-ended problem-solving work that combines design problems, decision-making problems, and troubleshooting problems, among other types [36]. To account for a progression in structuredness and complexity from the first to the last years, variable degrees of scaffolding and personalized feedback could be implemented instead of relying upon well-structured problems as is commonly done [7], [12], [69].

When instruction goes beyond the importance of solving a specific problem, assessment should focus on the problem-solving process engaged by the student rather than on the students' ability to recall knowledge or procedures. Consequently, students need feedback on their understanding of the problem and their ability to transfer knowledge, skills, and procedures from different types of problems to the problem they face. Additionally, feedback is needed on the cognitive skills the student should activate and prior domain knowledge to retrieve, based on the kind of problem that faces. Finally, students need feedback on their ability to construct arguments that support their solutions to problems [34].

As multiple forms of assessments are needed in complex problem-solving, instruments can also vary. The understanding of the problem type can be assessed from a problem schema. A problem schema allows students to understand the elements of the problems and how they interact as well as the procedures for solving the problem. Therefore, recognizing similar problems helps students identify the specific knowledge to retrieve, the skills they need to transfer to the novel problem and processes they should engage [34], [35].

The ability to transfer knowledge, skills, and procedures to solve a problem successfully is known as problemsolving performance. Assessment of solving-problem performance should include the capacity of students to construct a response, product, or solution and not just the selection of predefine alternatives or answers. Assessment of the quality of the proposed solution and assessing students' behaviour on the different tasks to generate the solution are also needed. Assessment and the corresponding feedback on students' problem-solving performance are commonly given using a rubric [12], [35].

Several cognitive skills are required to learn to solve problems depending on the type of problem. However, analogical reasoning and causal reasoning are fundamental cognitive skills required for the solution of all types of problems. Problem-solving is largely a process of understanding the causal relationship between problem elements and making inferences or predictions on the causes of specific states. Assessment and the corresponding feedback on analog and causal reasoning required elaborating constructed responses where problem elements and their relationships can be clearly stated. Constructed responses are open-ended students work in nature, and they can be communicated by different means. Responses to structured open-ended questionnaires range from written reports, oral dissertations, graphical representations of problem schemas, conceptual or mind maps, observation in class, and self-reflection 
questionnaires. At this point, it is important to note that Formulating short answers responses to guided statements or selecting from a set of standardized options will not be adequate to assess students' understanding of the problem. The present review focuses on text-based constructed responses to open-ended questions.

Similar to cognitive skills, domain knowledge is required to solve problems. In fact, prior knowledge has a significant impact on problem understanding and problemsolving performance [34]. However, because wellstructured problems have been broadly used in secondary and undergraduate education, assessment of domain knowledge has focused on the ability to recall conceptual or procedural knowledge rather than on the skill of transferring knowledge between different types of problems. Feedback is needed not only in knowledge correctness and relevance to solving a specific problem. Feedback is needed on the ability to synthesize knowledge for transferring to a novel situation, is needed.

Beyond analogical and causal reasoning, questioning strategies, problem modelling, argumentation, and metacognitive autoregulation, are relevant cognitive skills to CPS. Argumentation provided by the students on how and why they solved problems as they did is a powerful form of assessment. It provides insight into their problemsolving reasoning, especially for ill-structured problems [7]. Feedback on argumentative reasoning can aid in correcting students' misconceptions and improve their thinking and learning.

Under the analytical framework described above, this contribution explores if the existing literature in automated assessment or feedback for open-ended student work can provide meaningful feedback on complex problem-solving skills.

\section{LiteratURE ON AUTOMATED FEEDBACK FOR OPEN-ENDED WORK}

In the last ten years, a wide variety of different approaches using machine learning (ML) algorithms have been implemented to automate feedback to students' assessments. Automated feedback on constructed responses to open-ended, complex problems can be provided through intelligent tutor systems, automated scoring applications, and collaborative learning platforms [58]. This section summarizes relevant studies based on whether feedback on students' written inputs is given on cognitive skills and domain knowledge, on understanding the problem through problem schema assessment, on student problem-solving performance, or argumentation reasoning.

\subsection{Cognitive Skills and Domain Knowledge}

Cognitive skills like analogical and causal reasoning, problem modelling, and argumentation require identifying key concepts and the semantic relationship established by the student between these concepts. Cognitive skills like analogical, causal, and argumentative reasoning require identifying key concepts and the semantic relationship established by the student between these concepts. To the best of our knowledge, there is no automated system that gives feedback on CPS, specifically in the context of engineering education. Some research was found in assessing analogue reasoning based on concept maps [11], [26], [30] and on causal reasoning for essay argumentation [15], [27], [41], [43], [70].

Several studies score open-ended responses based on one or more features extracted from students' responses. Until the past few years, most automated assessment was based on conventional machine learning approaches. Learning algorithms such as support vector machine (SVM), Naïve Bayes classification, linear discriminant analysis (LDA), decision trees, artificial neural network (ANN), among others, depend heavily on engineering features. Features in conventional methods were based, for the most part, on conceptual domain knowledge. In contrast, deep learning approaches are based on representations that are not only constructed from the relationship between features and output, but also on extracting features automatically from the raw data itself $[6,17]$.

\section{Conventional Machine Learning Approaches}

Conventional algorithms trained on either supervised, unsupervised, and semi-supervised, have been used to (a) assign scores, (b) classify students' responses, (c) identify key learning goals or concepts, and (d) predict group project performance based on online discussions, linguistic features, and work patterns [64].

\section{Supervised Conventional ML}

Supervised algorithms required labelled data to train a model, infer and act accordingly to give a score, provide feedback, and/or adjust learning strategies. However, labelling data involves human scoring or classifying the student's responses, which is time-consuming and may limit the possibility of applying the same model to other contexts. Data is primarily obtained from the students' responses, like keywords, word frequency, and sentence structure. Although demographic, sociological, or sensor data from game interactions or questionnaires can be included in the modelling [14], it is not common in literature. Nakamura et al. [50] and Nehm et al. [52] are examples of studies based solely on text-dependent attributes extracted from constructed answers.

Selecting a specific algorithm is an iterative process dependent on the specific purpose (e.g. for scoring student work or clustering similar responses) and data available. Within the supervised learning approach, the most used algorithms are (a) linear regression, (b) SVM, (c) Sequential Minimal Optimisation (SMO), a particular case of SVM, (d) decision tree, and (e) Naïve Bayes. The platforms to implement the above algorithms will also 
depend on the projects' objectives and, to some extent, on developers' programming skills. Nehm and Haerting [53] used the text analysis tools in the commercial software SPSS to assess students' understanding of key concepts. Their study is based on a concept inventory and specific patterns between these concepts built by experts in the field. Although very good Cohen's Kappas were obtained, the building of the inventory and identification of respective patterns requires a significant time investment by experts. In addition, transferring the model to a different context is difficult and time-consuming.

To overcome these challenges, user-friendly platforms like c-rater-ML [42], LightSIDE [46, 47] and EvoGrader [49], [52] built using LightSIDE, have been developed. All of them rely on a supervised implementation of the algorithms mentioned above, though they vary on the degree of preprocessing, training, and/or validation required from the instructors. Notwithstanding, they may not require extensive time and computing resources. All of them have been extensively trained in the "back-end" and therefore are tied to some expert's corpus knowledge context. For example, EvoGrader, with a high machinehuman scoring agreement, does not require any training to provide automatic feedback in the field of evolutionary biology. Nonetheless, the back-end component of EvoGrader, was trained with 10,270 scored evolutionary explanations in response to 86 different predefined ACORNS (Assessing COntextual Reasoning about Natural Selection) instrument items.

\section{Non-supervised Conventional ML}

In contrast to supervised ML approaches, unsupervised ML does not require labelled data for the training process. Latent structures, distribution or patterns present in data are identified without relying on external expert labels. Although less frequently used, this approach can combine different types of attributes: demographic and academic. The few studies that have employed unsupervised ML in recent years have been restricted to (a) linear regression, (b) Bayesian network and (c) SVM algorithm to identify the relationships between variables and classify students' responses. This classification approach to aid in a posterior supervised scoring showed good to excellent Cohen's Kappas agreement in [63]. Although semi-supervised machine learning has the advantage of improving the accuracy of unsupervised models without the cost in time and data preparation, it is not common in literature. The application proposed by [37] to provide feedback to numerous programming assignments use semi-supervised learning. They cluster students' responses by solution approach and generate verified feedback based on the instructor validation of each approach. A similar approach was implemented for Intelligent Tutoring Systems (ITS) in [20].

\section{Deep Learning Approaches}

While conventional machine learning approaches are adequate for predicting scores for structured closed-end responses, they required extensive data preprocessing and exploratory analysis to identify the key features to model. As a result, the obtained models are very specific to the aiming task, the data used, and the handcraft representations extracted for training. Patterns in openended responses will likely be related to more abstract relationships between concepts and hierarchical patterns within these relationships. Deep learning methods have excelled in tasks where knowing which features should be extracted is difficult.

In this review, a few studies were identified, aiming to detect connections between key concepts or aim to group students' responses according to semantic similarities rather than lexical similarities. Although designed for science explanatory essay scoring, the structured learning approach using Recurrent Neural Networks (RNN) at the sentence and whole essay level is a promising future research area [29], [28], [59]. Other neural network models based on variations of RNN like LSTM [1], RNN with a co-attention module [65], or even convolution neural network [9] have been successfully implemented for automated essay scoring. Another promising study by Zhao [68] provides targeted feedback based on misconceptions in online learning environments using key phrase extraction and semantic embedding using different stateof-the-art Natural Language Process (NLP) techniques. Three types of text segmentation were used to extract key points: statistics-based key phrase extraction, graph-based key phrase extraction such as RAKE (Rapid Automatic Keyword Extraction [55]), and semantic-based key phrase extraction based on pre-trained language BERT (Bidirectional Encoder Representations from Transformers) models. Although, not directly develop for assessment of analogical reasoning, "The Retriever" tool develop by [21] for creative idea generation based on analogical reasoning and ontology could be explored to identify evidence of this type of reasoning in students work.

Most of the research review in this section is intended to assess content or domain knowledge in addition to cognitive skills. In general, the assessment of domain knowledge applied in these studies is based on the similarity classification of generic features to an experts' set of responses.

\subsection{Student Problem-Solving Performance}

Not all dimensions of student performance will be possible to assess from text-based constructed responses. Feedback on the student's performance to construct a response, product, or solution, and not just selecting predefined alternatives, can be included in the problem schema assessment. Performance-related to student behaviour will assess better through in-class observation or rubrics for self- and peer- reflection. Automated 
assessment will be beneficial considering that often reliability in applying a rubric can be an issue. Notwithstanding, Yoo and Kim [62] proposed a classification approach based on linguistic features, dialogue roles and work patterns in online discussion boards on engineering courses to predict group performance.

For the purposes of this review, those associated solely with the quality of the products, writing reports or openended constructed responses, such as spelling, or grammar automatic reviewers are not included. On the other hand, research on the quality of writing, such as sentence fluency, clarity, and coherence, has been researched in the past based on the classification of engineering features [45], [59]. Recent developments using deep learning methods, such as the one proposed by Mathias \& Bhattacharyya [45] based on a hierarchical Convolutional Neural Network and Long Short-Term Memory (CNN-LSTM) architecture or in attention-based Recurrent Convolutional Neural Network presented by Dong et al. [9], show promising results generalizing different aspects of writing quality for holistic grading and richer feedback.

Wiley [59] mentioned that other aspects for assessing the quality of scientific explanations, such as integration and transformation of the information, quality of sources, or completeness of the answer, have been approached through Latent Semantic Analysis (LSA, [40]). LSA has been implemented in Intelligent Tutoring Systems (ITS) such as AutoTutor and Meta Tutor. However, LSA and other similar approaches such as coherence index (CohMetrix, [48]) analysis are made at phrase or sentence level. Therefore, the authors [59] proposed a machine learning approach that combines the NLP system developed by Hughes [28] with LSA indices on similarity Coh-Metrix indices on coherence.

\subsection{Problem Schema}

One way to assess understanding of a problem is through problem schema. The quality of problem schema is predictive of problem-solving ability [34]. Building a schema of a problem is not an easy task. It requires analogical reasoning to compare the target problem to previous models. Recognition of elements, the relationship between them and possible solutions is an important form to assess problem-solving skills. Schemas for ill-structured would be less predictable, resulting in greater difficulty of assessment and therefore giving feedback on them.

Existing tools to assess conceptual or knowledge maps, like AKOVIA (Automated Knowledge Visualization and Assessment) develop by [30], are based on similarity metrics calculated from semantic analysis from written text or graphical representations and compared against single or multiple references. These metrics result in specific patterns in problem space. Although individuals may perceive problems differently and focus on different elements, domain experts' patterns have certain similarities that are not observed in novices. Based on dissimilarity in problem space patterns, assessing students' understanding of a problem and immediate feedback on their missing concepts or misconceptions could be given. On [11], a good agreement of AKOVIA results is reported compared against detailed qualitative assessment based on problemsolving rubrics (PSR).

Based on the same approach, [61] proposed adding information beyond identifying concepts at the nodes, including the match of prepositions that join concepts. This approach assumes that concepts at the map's nodes themselves can not detect the actual state of students' learning related to the elements of the problem unless a relationship between them is established. In particular, the authors proposed a weighted concept map as the criteria for assessment. Weights at the connecting prepositions are given by instructors and reflect the importance of specific relationships relative to others. Setting the weights at values between 0 and 1 allows for flexibility when students compared their models' metrics to experts' knowledge structures. Using this approach, Ho et al. [26] develop a knowledge-mapping assessment tool for medical education with high adherence of students.

\subsection{Argumentative Reasoning}

Argumentative reasoning has been pointed out by Jonassen as an essential form of reasoning in problem-solving, especially in solving an ill-structured complex problem. Giving feedback on the quality of the students' arguments promotes problem-solving. In addition, it provides insight into their problem-solving reasoning and constructed arguments provide evidence of the student learning process.

Originated from the scoring point of view, automated essay scoring (AES) has a vast tradition in machine learning and natural language processing [38]. Relevant to the assessment of CPS, recent developments in AES research [15] [27], [24], [29], [45], [65], [67], [68] have been motivated by increasing attention to developing argumentation skills in STEM education to deepen scientific and critical thinking. Deep learning architectures based on LSTM with or without attention, hierarchical $\mathrm{CNN}$, and the use of pre-train language models like BERT have successfully been able to extract high-order features associated with argumentation features. Particularly, [41], [66] are examples of automated feedback systems on argumentation skills of student work.

\section{CONCLUSIONS AND FUTURE RESEARCH}

Complex problem-solving skills are at the core of engineering education, yet assessing these skills is a complex task. In order to be an effective instructional strategy, formative feedback has to be meaningful and optimum in timing. In that sense, educational technologies can be a valuable supporting effective formative feedback 
beyond the fact that they can deliver feedback to large classes in a timely fashion. Automate feedback to openended student work can aid instructors to expose the students to a variety of increasingly complex authentic problems and provide tailored scaffolding.

However, providing feedback only on the ability of students to recall concepts or solutions will not help them gain the ability to know what knowledge and skills they need to apply in ill-structured complex problems prevalent in engineering practice. Under the proposed framework, to develop CPS, feedback should surpass domain knowledge and be provided in the student's understanding of the problem. The reasoning follows to solve the problem and the argumentation of those solutions.

Research on automated assessment using machine learning is vast. Several methods and applications have been developed using conventional ML approaches, and more recently, using deep learning algorithms. However, because feedback in open-ended student work involves identifying the relationship between conceptual knowledge, the acquisition of evidence in problem schemas, arguments, and reasoning requires identifying high-order features in the student writing. Deep learning approaches are advantageous in automatically revealing the inner structure between data and extracted high-level abstract features. In that sense, several interesting studies have been identified. Of particular interest, further research on the line of the work proposed by Gosh et al.,[15], Hughes [29], Mathias and Bhattacharyya [45], Zhang and Litman [65], [67], and Zhao et al. [68], will be pursued.

\section{Acknowledgements}

This work was supported by the DuPont Canada Chair in Engineering Education Research and Development.

\section{References}

[1] Dimitrios Alikaniotis, Helen Yannakoudakis, and Marek Rei, "Automatic Text Scoring Using Neural Networks," in Proceedings of the 54th Annual Meeting of the Association for Computational Linguistics (Volume 1: Long Papers), 2016, pp. 715-725.

[2] Susan A Ambrose, Michael W Bridges, Michele DiPietro, Marsha C Lovett, and Marie K Norman, How Learning Works: Seven Research-Based Principles for Smart Teaching. John Wiley \& Sons, 2010.

[3] John B Biggs, Teaching for Quality Learning at University: What the Student Does. McGraw-hill education (UK), 2011.

[4] Marilyn Binkley et al., "Defining Twenty-First Century Skills," in Assessment and Teaching of 21st Century Skills: Springer, 2012, pp. 17-66.

[5] Canadian Engineering Accreditation Board, Accreditation Criteria and Procedures. Ottawa, ON: Engineers Canada, 2017, p. 125.

[6] Nitin Kumar Chauhan and Krishna Singh, "A Review on Conventional Machine Learning Vs Deep Learning," in 2018 International Conference on Computing, Power and
Communication Technologies (GUCON), 2018: IEEE, pp. 347-352.

[7] Kyoo-Lak Cho and David H Jonassen, "The Effects of Argumentation Scaffolds on Argumentation and Problem Solving," Educational Technology Research and Development, vol. 50, no. 3, p. 5, 2002.

[8] B. Csapo and G. Molnar, "Potential for Assessing Dynamic Problem-Solving at the Beginning of Higher Education Studies," Frontiers in Psychology, vol. 8, p. 2022, 2017, doi: 10.3389/fpsyg.2017.02022.

[9] Fei Dong and Yue Zhang, "Automatic Features for Essay Scoring-an Empirical Study," in Proceedings of the 2016 conference on empirical methods in natural language processing, 2016, pp. 1072-1077.

[10] Kevin Dunbar, "Problem Solving," in A Companion to Cognitive Science, William Bechtel and George Graham Eds. Londo, England: Blackwell, 1998, pp. 289-298.

[11] Deniz Eseryel, Dirk Ifenthaler, and Xun Ge, "Validation Study of a Method for Assessing Complex Ill-Structured Problem Solving by Using Causal Representations," Educational Technology Research and Development, vol. 61, no. 3, pp. 443-463, 2013/06/01 2013, doi: 10.1007/s11423-013-9297-2.

[12] Brian Frank, Natalie Simper, and James Kaupp, "Formative Feedback and Scaffolding for Developing Complex Problem Solving and Modelling Outcomes," European Journal of Engineering Education, vol. 43, no. 4, pp. 552-568, 2018.

[13] Joachim Funke, "Complex Problem Solving: A Case for Complex Cognition?," Cognitive processing, vol. 11, no. 2, pp. 133-142, 2010.

[14] Ramla Ghali, Sébastien Ouellet, and Claude Frasson, "Lewispace: An Exploratory Study with a Machine Learning Model in an Educational Game," Journal of Education and Training Studies, vol. 4, no. 1, pp. 192-201, 2016.

[15] Debanjan Ghosh, Beata Beigman Klebanov, and Yi Song, "An Exploratory Study of Argumentative Writing by Young Students: A Transformer-Based Approach," arXiv preprint arXiv:2006.09873, 2020.

[16] Ilya Goldin, Susanne Narciss, Peter Foltz, and Malcolm Bauer, "New Directions in Formative Feedback in Interactive Learning Environments," International Journal of Artificial Intelligence in Education, vol. 27, no. 3, pp. 385392, 2017, doi: 10.1007/s40593-016-0135-7.

[17] Ian Goodfellow, Yoshua Bengio, Aaron Courville, and Yoshua Bengio, Deep Learning (no. 2). MIT press Cambridge, 2016.

[18] Samuel Greiff, Sascha Wüstenberg, Daniel V Holt, Frank Goldhammer, and Joachim Funke, "Computer-Based Assessment of Complex Problem Solving: Concept, Implementation, and Application," Educational Technology Research and Development, vol. 61, no. 3, pp. 407-421, 2013.

[19] Samuel Greiff, Andreas Fischer, Matthias Stadler, and Sascha Wüstenberg, "Assessing Complex Problem-Solving Skills with Multiple Complex Systems," Thinking \& Reasoning, vol. 21, no. 3, pp. 356-382, 2015.

[20] Sebastian Gross, Xibin Zhu, Barbara Hammer, and Niels Pinkwart, "Cluster Based Feedback Provision Strategies in Intelligent Tutoring Systems," presented at the Proceedings of the 11th international conference on Intelligent Tutoring Systems, Chania, Crete, Greece, 2012. [Online]. Available: https://doi.org/10.1007/978-3-642-30950-2_127. 
[21] Ji Han, Feng Shi, Liuqing Chen, and Peter R. N. Childs, "A Computational Tool for Creative Idea Generation Based on Analogical Reasoning and Ontology," Artificial Intelligence for Engineering Design, Analysis and Manufacturing, vol. 32, no. 4, pp. 462-477, 2018, doi: $10.1017 / \mathrm{s} 0890060418000082$.

[22] John Hattie and Helen Timperley, "The Power of Feedback," Review of educational research, vol. 77, no. 1, pp. 81-112, 2007.

[23] John Hattie, "The Applicability of Visible Learning to Higher Education," Scholarship of teaching and learning in psychology, vol. 1, no. 1, p. 79, 2015.

[24] Michael Heilman and Nitin Madnani, "The Impact of Training Data on Automated Short Answer Scoring Performance," in Proceedings of the Tenth Workshop on Innovative Use of NLP for Building Educational Applications, 2015, pp. 81-85.

[25] Christoph Nils Herde, Sascha Wüstenberg, and Samuel Greiff, "Assessment of Complex Problem Solving: What We Know and What We Don't Know," Applied Measurement in Education, vol. 29, no. 4, pp. 265-277, 2016, doi: 10.1080/08957347.2016.1209208.

[26] Veronica W Ho, Peter G Harris, Rakesh K Kumar, and Gary M Velan, "Knowledge Maps: A Tool for Online Assessment with Automated Feedback," Medical education online, vol. 23, no. 1, p. 1457394, 2018.

[27] Chenn-Jung Huang, Yu-Wu Wang, Tz-Hau Huang, YingChen Chen, Heng-Ming Chen, and Shun-Chih Chang, "Performance Evaluation of an Online Argumentation Learning Assistance Agent," Computers \& Education, vol. 57, no. 1, pp. 1270-1280, 2011, doi: 10.1016/j.compedu.2011.01.013.

[28] Simon Hughes, Peter Hastings, Mary Anne Britt, Patricia Wallace, and Dylan Blaum, "Machine Learning for Holistic Evaluation of Scientific Essays," in International Conference on Artificial Intelligence in Education, 2015: Springer, pp. 165-175.

[29] Simon Mark Hughes, "Automatic Inference of Causal Reasoning Chains from Student Essays," 2019.

[30] Dirk Ifenthaler, "Akovia: Automated Knowledge Visualization and Assessment," Technology, Knowledge and Learning, vol. 19, no. 1, pp. 241-248, 2014/07/01 2014, doi: 10.1007/s10758-014-9224-6.

[31] International Engineering Alliance, "Graduate Attributes and Professional Competencies," 2013. Accessed: Dec 13th, $2020 . \quad$ [Online]. Available: http://www.washingtonaccord.org/IEA-Grad-Attr-ProfCompetencies.pdf

[32] David Jonassen, Johannes Strobel, and Chwee Beng Lee, "Everyday Problem Solving in Engineering: Lessons for Engineering Educators," Journal of Engineering Education, vol. 95, no. 2, pp. 139-151, 2006.

[33] David H Jonassen, "Toward a Design Theory of Problem Solving," Educational Technology Research and Development, vol. 48, no. 4, pp. 63-85, 2000.

[34] David H Jonassen, Learning to Solve Problems: A Handbook for Designing Problem-Solving Learning Environments. Routledge, 2010.

[35] David H Jonassen, "Assessing Problem Solving," in Handbook of Research on Educational Communications and Technology: Springer, 2014, pp. 269-288.

CEEA-ACEG21; Paper 43

University of Prince Edward Island; June $21-23,2021-7$ of 8 -
[36] David H. Jonassen, "Engineers as Problem Solvers," in Cambridge Handbook of Engineering Education Research, Aditya Johri and Barbara M. Olds Eds.: Cambridge University Press, 2014, pp. 103-118.

[37] Shalini Kaleeswaran, Anirudh Santhiar, Aditya Kanade, and Sumit Gulwani, "Semi-Supervised Verified Feedback Generation," presented at the Proceedings of the 2016 24th ACM SIGSOFT International Symposium on Foundations of Software Engineering, Seattle, WA, USA, 2016. [Online]. Available: https://doi.org/10.1145/2950290.2950363.

[38] Zixuan Ke and Vincent Ng, "Automated Essay Scoring: A Survey of the State of the Art," in IJCAI, 2019, pp. 63006308.

[39] Avraham N Kluger and Angelo DeNisi, "The Effects of Feedback Interventions on Performance: A Historical Review, a Meta-Analysis, and a Preliminary Feedback Intervention Theory," Psychological bulletin, vol. 119, no. 2, p. 254, 1996.

[40] Thomas K. Landauer, Peter W. Foltz, and Darrell Laham, "An Introduction to Latent Semantic Analysis," Discourse Processes, vol. 25, no. 2-3, pp. 259-284, 1998, doi: 10.1080/01638539809545028.

[41] Hee-Sun Lee, Amy Pallant, Sarah Pryputniewicz, Trudi Lord, Matthew Mulholland, and Ou Lydia Liu, "Automated Text Scoring and Real-Time Adjustable Feedback: Supporting Revision of Scientific Arguments Involving Uncertainty," Science Education, vol. 103, no. 3, pp. 590622, 2019, doi: 10.1002/sce.21504.

[42] Ou Lydia Liu, Joseph A Rios, Michael Heilman, Libby Gerard, and Marcia C Linn, "Validation of Automated Scoring of Science Assessments," Journal of Research in Science Teaching, vol. 53, no. 2, pp. 215-233, 2016.

[43] Liyang Mao et al., "Validation of Automated Scoring for a Formative Assessment That Employs Scientific Argumentation," Educational Assessment, vol. 23, no. 2, pp. 121-138, 2018, doi: 10.1080/10627197.2018.1427570.

[44] Marina Marchisio, Alice Barana, Michele Fioravera, Sergio Rabellino, and Alberto Conte, "A Model of Formative Automatic Assessment and Interactive Feedback for Stem," in 2018 IEEE 42nd Annual Computer Software and Applications Conference (COMPSAC), 2018, vol. 1: IEEE, pp. 1016-1025.

[45] Sandeep Mathias and Pushpak Bhattacharyya, "Can Neural Networks Automatically Score Essay Traits?," in Proceedings of the Fifteenth Workshop on Innovative Use of NLP for Building Educational Applications, 2020, pp. 85-91.

[46] E Mayfield and C Rosé, "Side: The Summarization Ide (User's Manual)," ed: Carnegie Mellon University, 2010

[47] Elijah Mayfield and Carolyn Penstein Rosé, "Lightside: Open Source Machine Learning for Text," in Handbook of Automated Essay Evaluation: Current Applications and New Directions, Mark D. Shermis and Jill Burstein Eds.: Routledge, 2013, pp. 124-135.

[48] Danielle S McNamara, Arthur C Graesser, Philip M McCarthy, and Zhiqiang Cai, Automated Evaluation of Text and Discourse with Coh-Metrix. Cambridge University Press, 2014.

[49] Kayhan Moharreri, Minsu Ha, and Ross $\mathrm{H}$ Nehm, "Evograder: An Online Formative Assessment Tool for Automatically Evaluating Written Evolutionary Explanations," Evolution: Education and Outreach, vol. 7, no. 1, pp. 1-14, 2014. 
[50] Christopher M. Nakamura, Sytil K. Murphy, Michael G. Christel, Scott M. Stevens, and Dean A. Zollman, "Automated Analysis of Short Responses in an Interactive Synthetic Tutoring System for Introductory Physics," Physical Review Physics Education Research, vol. 12, no. 1, p. 010122, 03/16/ 2016, doi: 10.1103/PhysRevPhysEducRes.12.010122.

[51] National Research Council, How People Learn: Brain, Mind, Experience, and School: Expanded Edition. National Academies Press, 2000.

[52] Ross $\mathrm{H}$ Nehm, Minsu $\mathrm{Ha}$, and Elijah Mayfield, "Transforming Biology Assessment with Machine Learning: Automated Scoring of Written Evolutionary Explanations," Journal of Science Education and Technology, vol. 21, no. 1, pp. 183-196, 2012.

[53] Ross H Nehm and Hendrik Haertig, "Human Vs. Computer Diagnosis of Students' Natural Selection Knowledge: Testing the Efficacy of Text Analytic Software," Journal of Science Education and Technology, vol. 21, no. 1, pp. 56-73, 2012.

[54] Carolyn P Rosé, Elizabeth A McLaughlin, Ran Liu, and Kenneth R Koedinger, "Explanatory Learner Models: Why Machine Learning (Alone) Is Not the Answer," British Journal of Educational Technology, vol. 50, no. 6, pp. 29432958, 2019.

[55] Stuart Rose, Dave Engel, Nick Cramer, and Wendy Cowley, "Automatic Keyword Extraction from Individual Documents," Text Mining: Applications and Theory, vol. 1, pp. 1-20, 2010.

[56] Shirley W Schiever and Carol June Maker, "Enrichment and Acceleration: An Overview and New Directions," Handbook of Gifted Education, vol. 2, pp. 113-125, 1997.

[57] Valerie J Shute, "Focus on Formative Feedback," Review of educational research, vol. 78, no. 1, pp. 153-189, 2008.

[58] J Michael Spector et al., "Technology Enhanced Formative Assessment for 21st Century Learning," 2016.

[59] Jennifer Wiley et al., "Different Approaches to Assessing the Quality of Explanations Following a Multiple-Document Inquiry Activity in Science," International Journal of Artificial Intelligence in Education, vol. 27, no. 4, pp. 758790, 2017.

[60] Dylan Wiliam, "The Role of Formative Assessment in Effective Learning Environments," in The Nature of Learning: Using Research to Inspirre Practice, Hanna Dumont, David Istance, and Francisco Benavides Eds., (Educational Research and Innovation: OECD Publishing, 2010, pp. 135-159.

[61] Po-Han Wu, Gwo-Jen Hwang, Marcelo Milrad, Hui-Ru Ke, and Yueh-Min Huang, "An Innovative Concept Map Approach for Improving Students' Learning Performance with an Instant Feedback Mechanism," British Journal of Educational Technology, vol. 43, no. 2, pp. 217-232, 2012.
[62] Jaebong Yoo and Jihie Kim, "Can Online Discussion Participation Predict Group Project Performance? Investigating the Roles of Linguistic Features and Participation Patterns," International Journal of Artificial Intelligence in Education, vol. 24, no. 1, pp. 8-32, 2014, doi: 10.1007/s40593-013-0010-8.

[63] Fabian Zehner, Christine Sälzer, and Frank Goldhammer, "Automatic Coding of Short Text Responses Via Clustering in Educational Assessment," Educational and Psychological Measurement, vol. 76, no. 2, pp. 280-303, 2016, doi: 10.1177/0013164415590022.

[64] Xiaoming Zhai, Yue Yin, James W. Pellegrino, Kevin C. Haudek, and Lehong Shi, "Applying Machine Learning in Science Assessment: A Systematic Review," Studies in Science Education, vol. 56, no. 1, pp. 111-151, 2020, doi: 10.1080/03057267.2020.1735757.

[65] Haoran Zhang and Diane Litman, "Co-Attention Based Neural Network for Source-Dependent Essay Scoring," in Proceedings of the Thirteenth Workshop on Innovative Use of NLP for Building Educational Applications, 2018, pp. 399-409.

[66] Haoran Zhang et al., "Erevise: Using Natural Language Processing to Provide Formative Feedback on Text Evidence Usage in Student Writing," in Proceedings of the AAAI Conference on Artificial Intelligence, 2019, vol. 33, no. 01, pp. 9619-9625.

[67] Haoran Zhang and Diane Litman, "Automated Topical Component Extraction Using Neural Network Attention Scores from Source-Based Essay Scoring," in Proceedings of the 58th Annual Meeting of the Association for Computational Linguistics, 2020, pp. 8569-8584.

[68] Jinjin Zhao, Kim Larson, Weijie Xu, Neelesh Gattani, and Candace Thille, "Targeted Feedback Generation for Constructed-Response Questions," 2020.

[69] Wei Zheng and Mr Yanhua Cao, "Implementation and Outcomes of Scaffolding Cyber-Enabled Collaborative Learning in Multiple Stem Courses," presented at the 122nd ASEE Annual Conference, Seattle, WA, 2015. [Online]. Available: https://www.asee.org/public/.

[70] Mengxiao Zhu, Hee-Sun Lee, Ting Wang, Ou Lydia Liu, Vinetha Belur, and Amy Pallant, "Investigating the Impact of Automated Feedback on Students' Scientific Argumentation," International Journal of Science Education, vol. 39, no. 12, pp. 1648-1668, 2017, doi: 10.1080/09500693.2017.1347303. 